University of Nebraska - Lincoln

DigitalCommons@University of Nebraska - Lincoln

May 1979

\title{
Magnetic properties of iron-doped layer-structure dichalcogenides
}

S.J. Hillenius

Department of Physics, University of Virginia, Charlottesville, Virginia

R.V. Coleman

Department of Physics, University of Virginia, Charlottesville, Virginia

E.R. Domb

University of Nebraska - Lincoln

David J. Sellmyer

University of Nebraska-Lincoln, dsellmyer@unl.edu

Follow this and additional works at: https://digitalcommons.unl.edu/physicssellmyer

Part of the Physics Commons

Hillenius, S.J.; Coleman, R.V.; Domb, E.R.; and Sellmyer, David J., "Magnetic properties of iron-doped layerstructure dichalcogenides " (1979). David Sellmyer Publications. 169.

https://digitalcommons.unl.edu/physicssellmyer/169

This Article is brought to you for free and open access by the Research Papers in Physics and Astronomy at DigitalCommons@University of Nebraska - Lincoln. It has been accepted for inclusion in David Sellmyer Publications by an authorized administrator of DigitalCommons@University of Nebraska - Lincoln. 


\title{
Magnetic properties of iron-doped layer-structure dichalcogenides
}

\author{
S. J. Hillenius and R. V. Coleman \\ Department of Physics, University of Virginia, \\ Charlottesville, Virginia 22901 \\ E. R. Domb and D. J. Sellmyer \\ Behlen Laboratory of Physics, University of Nebraska, \\ Lincoln, Nebraska 68588 \\ (Received 18 August 1978)
}

\begin{abstract}
Magnetic-susceptibility measurements have been made on dilute excess $\mathrm{Fe}$ alloys of the layer-structure crystals $\mathrm{TaSe}_{2}, \mathrm{NbSe}_{2}$, and $\mathrm{TaS}_{2}$. The $2 \mathrm{H}$ selenides show local-moment formation and obey a Curie-Weiss law in the range $300-30 \mathrm{~K}$ with effective moments in the range (2.5-4.5) $\mu_{\mathrm{B}}$. For $2 \mathrm{H}-\mathrm{Fe}_{x} \mathrm{TaSe}_{2}$ a strong susceptibility maximum is observed at low temperature and at low fields the maximum has a cusplike shape characteristic of a "spin-glass" type transition. The temperature of the maximum scales approximately linearly with $\mathrm{Fe}$ concentration and reaches $13 \mathrm{~K}$ for $x=0.10$. The presence of the magnetic impurities decreases the onset temperature of the charge-density wave and smears the transition. At the same time the magnetic impurities can stabilize a spin-density wave and the data are reviewed in terms of such a possibility. Crystals of $2 \mathrm{H}-\mathrm{Fe}_{x} \mathrm{TaS}_{2}$ do not show local-moment formation and the susceptibility measurements show a smearing of the charge-density wave but no depression of the onset temperature up to $x=0.05$. The Fe-doped $4 \mathrm{Hb}$ phases of $\mathrm{TaS}_{2}$ and $\mathrm{TaSe}_{2}$ show distinctly different behavior with $4 \mathrm{Hb}$ - $\mathrm{Fe}_{x} \mathrm{TaS}_{2}$ showing a strong-local-moment formation and a susceptibility maximum while $4 \mathrm{Hb}$ - $\mathrm{Fe}_{x} \mathrm{TaSe}_{2}$ shows only a weak-local-moment formation and no magnetic anomalies. The susceptibility behavior of the various phases also correlates with magnetic anomalies observed in the transport properties of the Fe-doped layer structures and these correlations will also be reviewed.
\end{abstract}

\section{INTRODUCTION}

Dilute iron alloys of the layer compounds $\mathrm{NbSe}_{2}$, $\mathrm{TaSe}_{2}$, and $\mathrm{TaS}_{2}$ have been studied using measurements of magnetoresistance, Hall effect, and magnetic susceptibility. When the compounds are doped with excess iron in the range $0.01<x<0.10$ to produce crystals of the form $\mathrm{Fe}_{x} M X_{2}$ the electronic properties are modified in different ways depending on the specific compound and phase. The $2 \mathrm{H}$ phases of the selenides generally show strong magnetic effects at low temperature with anomalous features in the transport properties ${ }^{1-4}$ and susceptibility. These include resistance minima and maxima, negative magnetoresistance, anomalous $\mathrm{Hall}$ effects and maxima in susceptibility. In contrast $2 \mathrm{H}-\mathrm{TaS}_{2}$ crystals when doped with excess iron show an increased superconducting transition temperature, an enhanced superconducting anisotropy, ${ }^{1.5}$ and no magnetic effects in either transport or susceptibility. The complete set of measurements allows a correlation of the electronic behavior with the charge-density-wave (CDW) transitons ${ }^{6}$ and the modifications produced by the impurities. While features such as the resistance minima can be explained by spin-flip Kondo-type scattering other features such as the susceptibility and resistivity maxima imply the possible onset of a spin-density wave (SDW) stabilized by the magnetic impurities.

The susceptibilities and transport properties of $4 H b-\mathrm{Fe}_{x} \mathrm{TaS}_{2}$ and $4 \mathrm{Hb}-\mathrm{Fe}_{x} \mathrm{TaSe}_{2}$ also show a variety of electronic changes induced by excess iron doping. Strong local-moment behavior and susceptibility maxima are observed in $4 \mathrm{Hb}$ - $\mathrm{Fe}_{x} \mathrm{TaS}_{2}$ while in $4 \mathrm{Hb}$ -

$\mathrm{Fe}_{x} \mathrm{TaSe}_{2}$ neither the transport properties nor susceptibility exhibit the features associated with magnetic scattering or stabilization of a SDW. This difference in electronic behavior between the two $4 \mathrm{Hb}$ phases is the reverse of that observed in the $2 \mathrm{H}$ phases of the same two compounds and suggests a sensitive dependence of the electronic structure and local-moment formation on the crystal-field environment of the $\mathrm{Fe}$ atom.

In this paper we emphasize the results of susceptibility measurements since a detailed analysis of the transport properties has previously been published. ${ }^{1}$ However new transport data on some phases and sufficient transport data on all phases and compounds will be included in order to establish the appropriate

4711 (1979 The American Physical Society 
correlations with the susceptibility data.

The susceptibility data on $2 \mathrm{H}-\mathrm{Fe}_{x} \mathrm{TaSe}_{2}$ have been developed in most detail in order to explore further the possible explanation for the unusual magnetic effects observed. Antoniou and Cohen ${ }^{7}$ have recently developed a theory which relates most of these magnetic properties to destabilization of the CDW and stabilization of a SDW by the magnetic impurities. This leads to a new kind of spin-glass which they call a "spin-density-wave glass." The theory has been developed in the context of a Landau mean-field theory and a detailed treatment ${ }^{8}$ is contained in a paper by Antoniou.

Fermi-surface measurements on a number of phases of $\mathrm{TaS}_{2}$ and $\mathrm{TaSe}_{2}$ have recently been reported by Hillenius and Coleman ${ }^{9}$ and the results show definite differences in the detailed Fermi-surface structure of the CDW state in the various materials and phases. These results will be reviewed briefly in order to consider any possible correlation with the observed differences in electronic behavior. induced by doping with excess iron.

\section{EXPERIMENTAL TECHNIQUES}

The single crystals used in these experiments were grown by the method of iodine-vapor transport. The starting materials mixed in powder form were sintered in vacuum at $950^{\circ} \mathrm{C}$ for five days to produce a free flowing powder of the appropriate iron alloy. Approximately three grams of powder were then sealed in a quartz tube with sufficient iodine to produce about one atmosphere at the growth temperature. The tube was then heated to the appropriate growth temperature and a temperature gradient was set with a programmed temperature controller.

The crystalline phases of the various powders and single crystals were checked by $\mathrm{x}$-ray powder patterns and by $x$-ray precession photographs. The iron concentrations in the powders and in the single crystals were checked for selected alloys with a spark mass spectrometer. Details of the growth parameters used to obtain single crystals of the various phases used in these experiments can be found in Ref. 1 .

For crystals of $\mathrm{NbSe}_{2}$ and $\mathrm{TaS}_{2}$ doped with high excess iron concentrations $(x \geqslant 0.26) \mathrm{x}$-ray data ${ }^{10,11}$ has shown the iron to be intercalated between the layers in the octahedral holes. This has not been checked directly for the low excess iron concentrations studied in the present experiments. However small concentrations of substitutional iron $x \geqslant 0.001$ are observed to stabilize the $1 T$ phase and this does not occur for crystal growth with excess iron up to $x=0.10$. The characteristic transport and susceptibility features such as minima and maxima all scale monotonically with iron concentration and the results of many experimental measurements with $x$ in the range $0-0.10$ suggest that a reliable systematic variation of iron concentration has been obtained. Some inhomogeneity in iron concentration can be expected particularly at low concentrations but the major features in both susceptibility and transport do not appear to be associated with other than a systematic variation of the intercalated iron concentration.

Susceptibility measurements were made using Faraday balances ${ }^{12}$ at both the University of Virginia and at the University of Nebraska. Measurements at Virginia were made on single crystals in a quartz sample holder with fields ranging between 0.5 and $4.5 \mathrm{kG}$. Temperature was controlled and measured between 1.5 and $300 \mathrm{~K}$ using resistance thermometers. The measurements at Nebraska were made on powders and stacked single crystals placed in sample holders made of quartz and Lexan. The temperature was varied between 1.5 and $300 \mathrm{~K}$ and magnetic fields up to $10.5 \mathrm{kG}$ were applied. Samples having masses in the range $10-30 \mathrm{mg}$ were used for the susceptibility measurements.

The temperature dependence of the resistivity and the Hall effect were made using standard four lead dc techniques with the current parallel to the layers. The samples were cleaved into thin sections and mounted using silver paint contacts. Typical sample dimensions were $0.5 \times 4 \mathrm{~mm}^{2}$ for the resistance measurement and $4 \times 8 \mathrm{~mm}^{2}$ for the Hall measurements. Sample thicknesses ranged from 20 to 200 $\mu \mathrm{m}$. The measurements were made in fields up to 70 $\mathrm{kG}$ using a superconducting solenoid. The temperature dependence of resistance and Hall effect were made using variable temperature sample holders operated with resistance heaters and helium exchange gas. Temperature was controlled and measured using both resistance and capacitance thermometers.

\section{EXPERIMENTAL RESULTS}

For excess iron doping in the range $0-10$ at. \% the $2 \mathrm{H}$ phases of $\mathrm{NbSe}_{2}$ and $\mathrm{TaSe}_{2}$ both show a resistance minimum behavior at low temperature and the susceptibilities can be fit to the Curie-Weiss expression $\chi(T)=\dot{\chi}_{0}+C /(T-\Theta)$ over most of the temperature range. $\theta$ is a characteristic constant called the Weiss temperature and $C$ is the Curie constant to be described in more detail in Sec. IV. A typical normalized resistivity plot for $2 \mathrm{H}$ - $\mathrm{Fe}_{x} \mathrm{TaSe}_{2}$ as a function of temperature is shown in Fig. 1. The resistivity shows a minimum between 10 and $20 \mathrm{~K}$ and a logarithmic rise below this minimum. At still lower temperatures a resistance maximum is also observed in the $2 H-\mathrm{Fe}_{x} \mathrm{TaSe}_{2}$ crystals. Below this maximum the resistance drops rapidly in zero magnetic field while for very high applied fields on the order of $150 \mathrm{kG}$ the resistance drop can be completely quenched. 


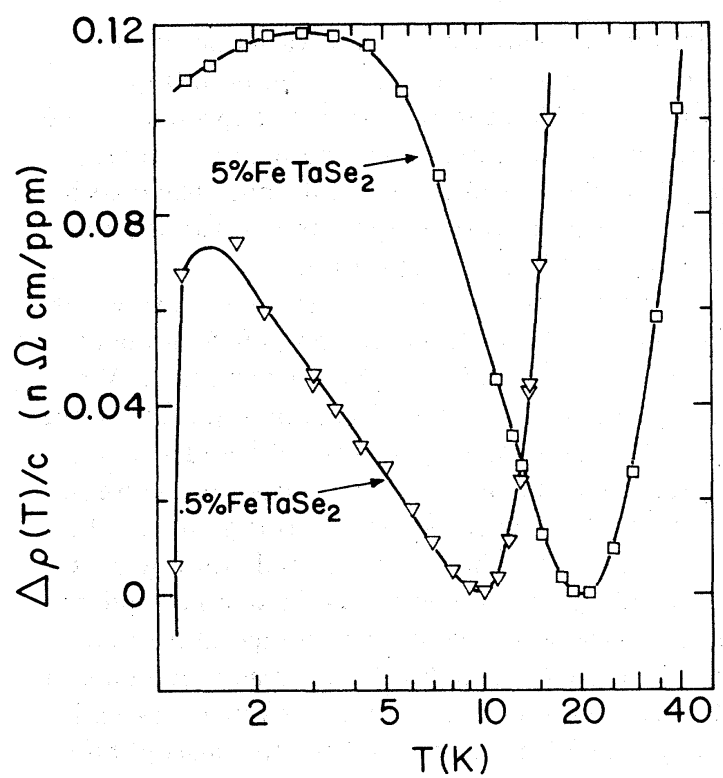

FIG. 1. Resistance minimum and maximum observed in $2 \mathrm{H}-\mathrm{TaSe}_{2}$ doped with excess $\mathrm{Fe}$ for $\mathrm{Fe}$ concentrations (c) of 0.5 at. $\%$ and 5 at. \%. (From Ref. 1).
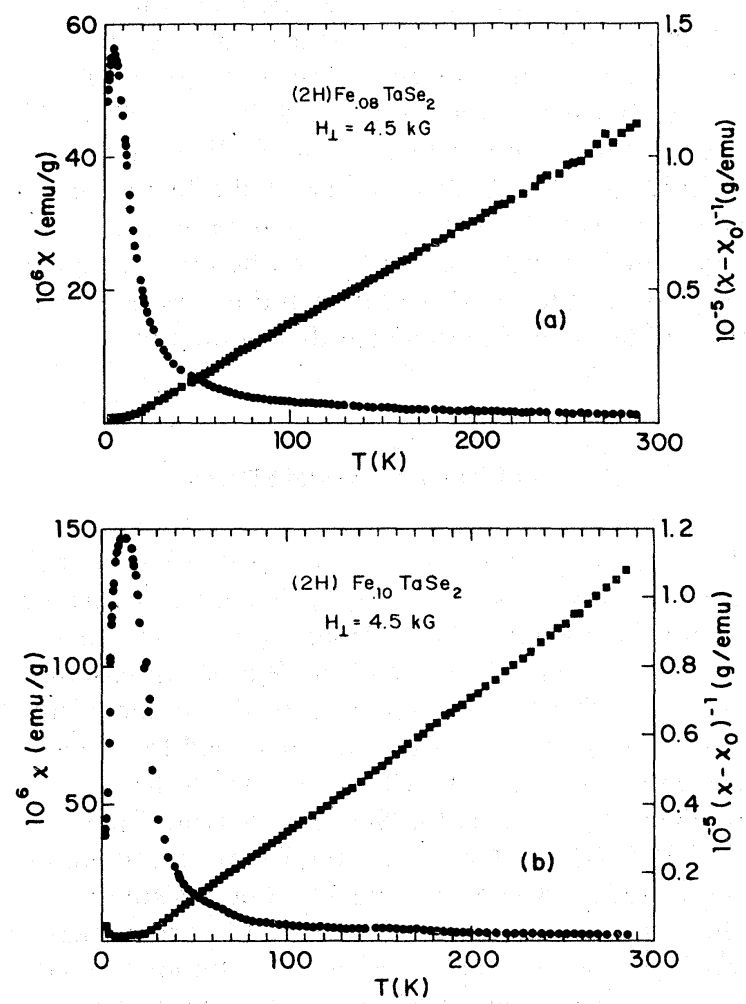

FIG. 2. Curie-Weiss susceptibility and susceptibility maximum observed in $2 \mathrm{H}-\mathrm{Fe}_{x} \mathrm{TaSe}_{2}$. Temperature range of measurement is $1.5-300 \mathrm{~K}$ and $1 / x$ vs temperature curves are also shown. (a) $x=0.08$. (b) $x=0.10$.

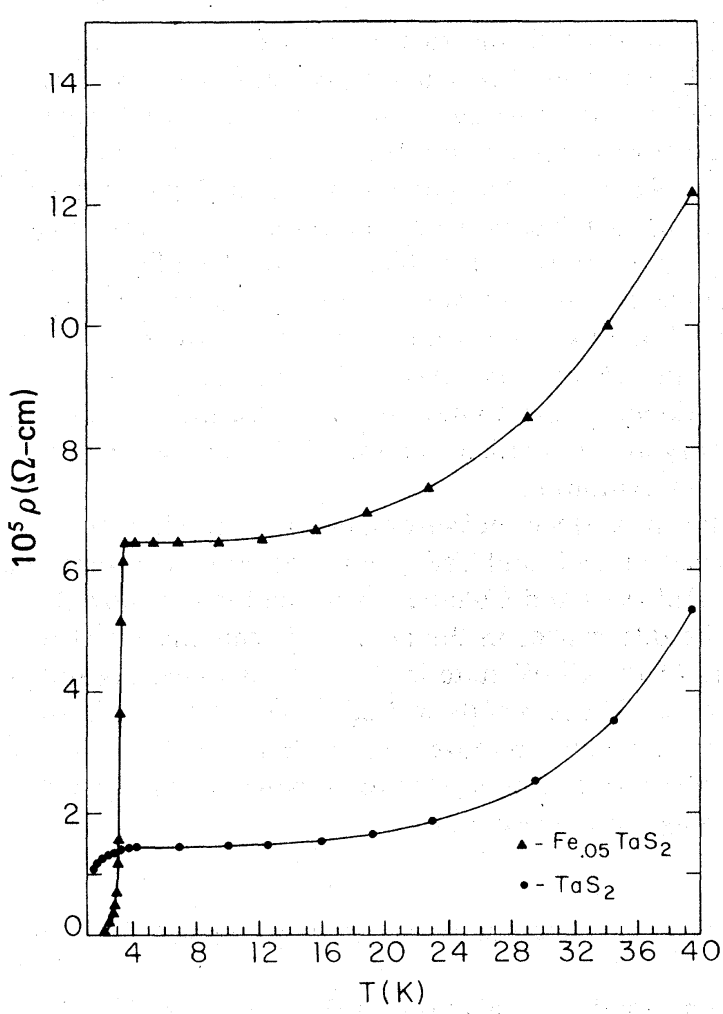

FIG. 3. Resistivity vs temperature for $2 \mathrm{H}-\mathrm{TaS}_{2}$ and for $2 H-\mathrm{Fe}_{0.05} \mathrm{TaS}_{2}$. The increase in superconducting transition temperature and residual resistance due to the Fe doping are clearly shown.

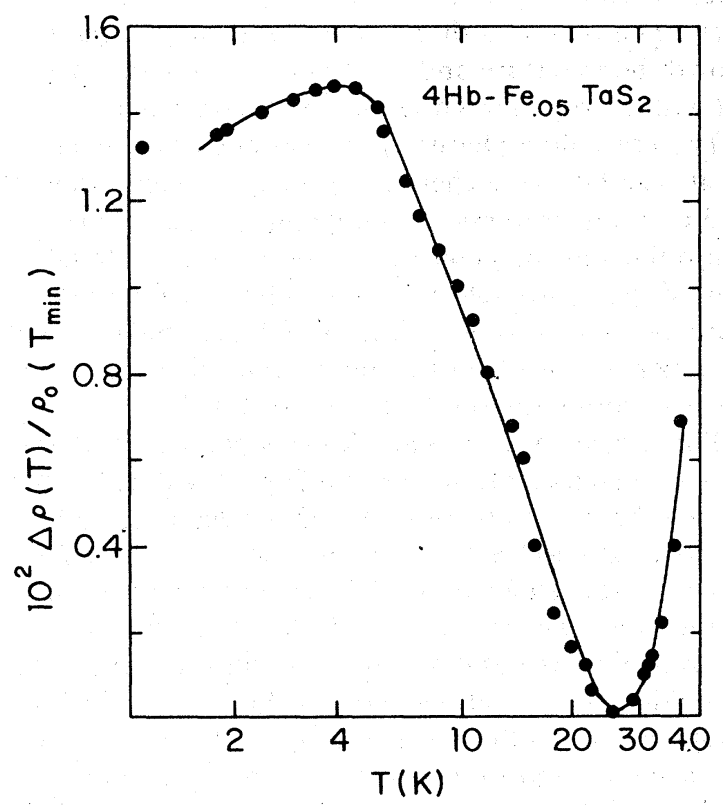

FIG. 4. Resistance minimum and maximum observed in $4 \mathrm{Hb}-\mathrm{TaS}_{2}$ due to doping with 5 at. $\% \mathrm{Fe}$. 
TABLE I. Susceptibility data for $\mathrm{Fe}_{x} M \mathrm{Se}_{2}$ compounds.

\begin{tabular}{cccccc}
\hline \hline Material & Phase & $\begin{array}{c}p_{\text {eff }} \\
\left(\mu_{\mathrm{B}}\right)\end{array}$ & $\begin{array}{c}\Theta \\
(\mathrm{K})\end{array}$ & $\begin{array}{c}x_{0} \\
\left(10^{-6} \mathrm{emu} / \mathrm{g}\right)\end{array}$ & $\begin{array}{c}T_{\max } \\
(\mathrm{K})\end{array}$ \\
\hline $\mathrm{Fe}_{0.005} \mathrm{TaSe}_{2}$ & $2 H$ (crystal) & $1.5 \pm 0.2$ & $-1 \pm 1$ & $0.60 \pm 0.05$ & $\ldots$ \\
$\mathrm{Fe}_{0.02} \mathrm{TaSe}_{2}$ & $2 H$ (crystal) & 1.25 & -2 & 0.58 & $2.0(4.5 \mathrm{kG})$, \\
$\mathrm{Fe}_{0.05} \mathrm{TaSe}_{2}$ & $2 H$ (crystal) & 2.9 & -1 & 0.65 & $2.6(0.41 \mathrm{kG})$ \\
& & & & & $4.7(4.5 \mathrm{kG})$ \\
$\mathrm{Fe}_{0.08} \mathrm{TaSe}_{2}$ & $2 H$ (crystal) & 2.8 & 7 & 0.65 & $5.6(0.45 \mathrm{kG})$ \\
$\mathrm{Fe}_{0.10} \mathrm{TaSe}_{2}$ & $2 H$ (crystal) & 3.6 & 21 & 0.60 & $12(4.5 \mathrm{kG})$ \\
$\mathrm{Fe}_{0.05} \mathrm{NbSe}_{2}$ & $2 H$ (crystal) & 3.0 & -6 & 1.06 & $13(0.6 \mathrm{kG})$ \\
$\mathrm{Fe}_{0.05} \mathrm{NbSe}_{2}$ & $2 H$ (powder) & 3.2 & -7 & 1.0 & $\cdots$ \\
\hline \hline
\end{tabular}

The susceptibility of the $2 H-\mathrm{Fe}_{x} \mathrm{TaSe}_{2}$ crystals also exhibits a maximum at temperatures in the range below $10 \mathrm{~K}$ as shown for the $2 \mathrm{H}-\mathrm{Fe}_{0.08} \mathrm{TaSe}_{2}$ crystal in Fig. 2(a) for data taken in a field of $1.5 \mathrm{kG}$. Strong magnetic fields reduce the susceptibility maximum and shift it to lower temperatures. The temperature of the maxima in both resistivity and susceptibility scales up with increased iron concentration and reaches approximately $13 \mathrm{~K}$ for the $2 H-\mathrm{Fe}_{0.10} \mathrm{TaSe}_{2}$ crystal as shown in Fig. 2(b).

The $2 H-\mathrm{Fe}_{x} \mathrm{NbSe}_{2}$ crystals show a similar resistance minimum below $20 \mathrm{~K}$ while no resistance maximum has been observed although it could occur below the lowest measuring temperature of $\sim 1.5 \mathrm{~K}$. For both $2 H-\mathrm{Fe}_{x} \mathrm{TaSe}_{2}$ and $2 H-\mathrm{Fe}_{x} \mathrm{NbSe}_{2}$ crystals the resistance minima can be completely quenched for magnetic fields of $150 \mathrm{kG}$ applied parallel to the layers. The Hall effect in both iron-doped $\mathrm{NbSe}_{2}$ and $\mathrm{TaSe}_{2} 2 \mathrm{H}$ phases shows an anomalous magnetic scattering term $^{1,13}$ at low temperature. This term is positive over the entire temperature range and rapidly dominates the negative Hall contribution arising from the pure CDW phase at low temperatures. Selected data on both the temperature and field dependence of the Hall effect will be given in Sec. III E.

In contrast with $2 \mathrm{H}$-TaSe${ }_{2}$ and $2 \mathrm{H}$ - $\mathrm{NbSe}_{2}$, excess iron doping of the $2 \mathrm{H}$-TaS crystals does not produce local-moment behavior and the transport properties show no evidence of magnetic scattering, but do show greatly enhanced superconducting properties. ${ }^{1,5}$ Figure 3 shows the resistivity versus temperature data for a $2 H-\mathrm{Fe}_{0.05} \mathrm{TaS}_{2}$ crystal and compares the behavior with that of the pure crystal. The superconducting transition temperature has increased from 0.8 to $3.5 \mathrm{~K}$ and a large increase in residual resistivity is observed. However no resistance minimum or other features associated with magnetic scattering are observed. These striking differences between the selenide and the sulfide $2 \mathrm{H}$ phase crystals doped with excess iron also correlate with the suppression of the CDW transition. Both susceptibility and Hall data relating to the CDW transition will be presented below.

In the case of $4 \mathrm{Hb}-\mathrm{Fe}_{x} \mathrm{TaS}_{2}$ both a resistance minimum and a resistance maximum are observed as shown in Fig. 4. These features behave in the same way as observed for $2 \mathrm{H}-\mathrm{Fe}_{x} \mathrm{TaSe}_{2}$ and the susceptibility again shows a maximum at low temperature which can be associated with the onset of an SDW. The $4 H b$ - $\mathrm{Fe}_{x} \mathrm{TaSe}_{2}$ crystals can only be grown with $x$ in the range $0-0.2$ and in this range of $\mathrm{Fe}$ concentration no magnetic effects have been observed in the transport properties and only a weak moment has been observed in the susceptibility measurements.

The above summary describes most of the transport results on the various phases and compounds and the susceptibility results show a consistent correlation with the differences in transport behavior. The following sections describe the detailed susceptibility measurements for each compound and phase.

\section{A. Susceptibility of $2 H-\mathrm{Fe}_{x} \mathrm{TaSe}_{2}$}

The pure $2 H$ phase of $\mathrm{TaSe}_{2}$ has a second-order normal to incommensurate CDW transition ${ }^{14}$ at $-122 \mathrm{~K}$ followed by a transition to a commensurate state at $\sim 90 \mathrm{~K}$. Systematic susceptibility measurements for $2 H$ - $\mathrm{Fe}_{x} \mathrm{TaSe}_{2}$ crystals with $x$ in the range $0-0.10$ have been made in the temperature range $1.5-300 \mathrm{~K}$. All of the $\mathrm{Fe}$ doped crystals in the above concentration range show a Curie-Weiss behavior and analysis of the $\chi$ vs $T$ plots has been used to determine values of the Curie constant $C$, the Weiss temperature $\theta$, and the effective moment

$p_{\text {eff }}=g\left[S(S+1]^{1 / 2}\right.$. As shown in Fig. 2 for the $x=0.08$ and $x=0.10$ crystals the $\chi$ vs $1 / T$ plots give 


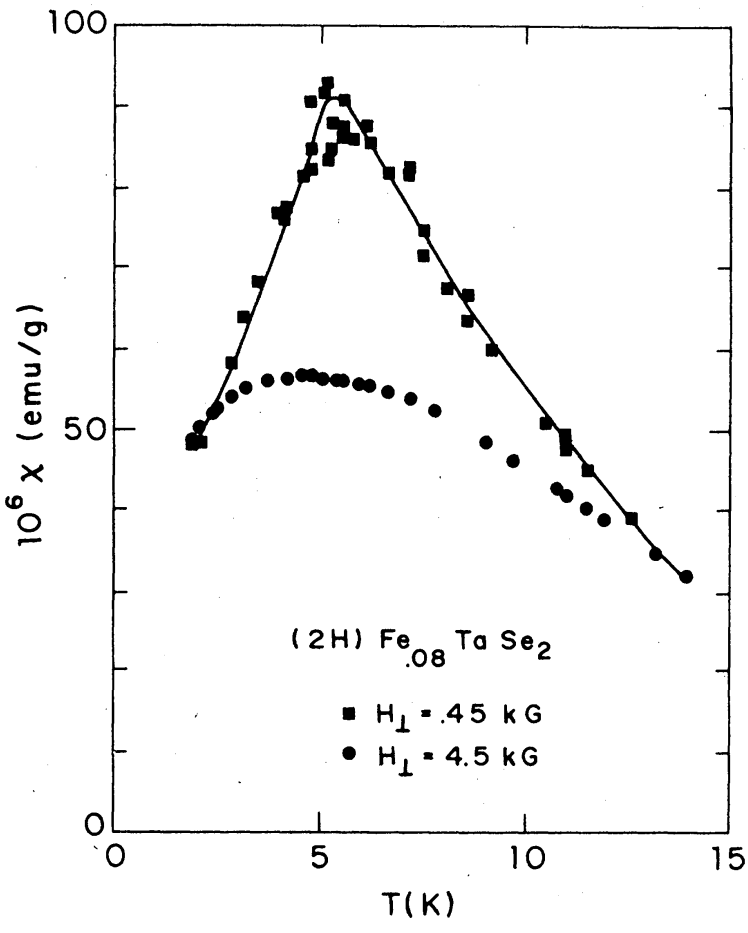

FIG. 5. Response of the susceptibility maximum in $2 \mathrm{H}$ $\mathrm{Fe}_{0.08} \mathrm{TaSe}_{2}$ to low applied magnetic fields. The cusplike form of the susceptibility maximum at low fields is characteristic of a spin-glass type of transition. The maximum becomes rounded and is depressed to lower temperatures at higher magnetic fields and is consistent with the onset of a spin-density wave.

reasonably straight lines and calculated values of $p_{\text {eff }}$ are 2.75 and $3.6 \mu_{\mathrm{B}}$ corresponding to spin values somewhat less than $\frac{3}{2}$ which would give $p_{\text {eff }}=3.9$. Susceptibility maxima occur at 5 and $13 \mathrm{~K}$ for $x=0.08$ and $x=0.10$, respectively. The presence of these maxima cause deviations in the Curie-Weiss behavior at low temperature and therefore influence the values of the calculated constants. The values of the constants listed in Table I are obtained by eliminating data points at the low-temperature end until successive calculation gives only a small change in $p_{\text {eff. }}$ For the higher $\mathrm{Fe}$ concentration range this condition is satisfied for temperatures above $20-30 \mathrm{~K}$.

The susceptibility maxima are relatively insensitive to magnetic fields from 1 to $5 \mathrm{kG}$ and are substantially depressed only for fields above $10 \mathrm{kG}$. However, the detailed shape of the maximum is quite sensitive at fields below $1 \mathrm{kG}$. For example as shown in Fig. 5 for the $\mathrm{Fe}_{0: 08} \mathrm{TaSe}_{2}$ crystal at $H_{1}=0.45 \mathrm{kG}$ the maximum has a cusplike shape while it becomes quite rounded at $H_{\perp}=4.5 \mathrm{kG}$. The cusplike behavior is expected for the onset of a spin-density wave (SDW) and this will be discussed in Sec. IV.

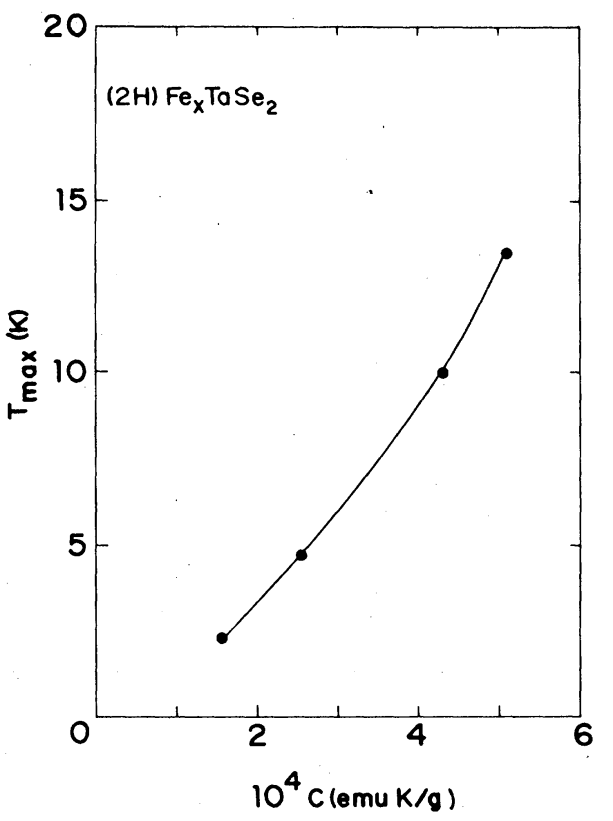

FIG. 6. Temperature of the susceptibility maximum in $2 \mathrm{H}-\mathrm{Fe}_{x} \mathrm{TaSe}_{2}$ as a function of measured Curie constant. The measured Curie constant also scales monotonically with the initial iron concentration of the sintered powder used to grow the crystals.

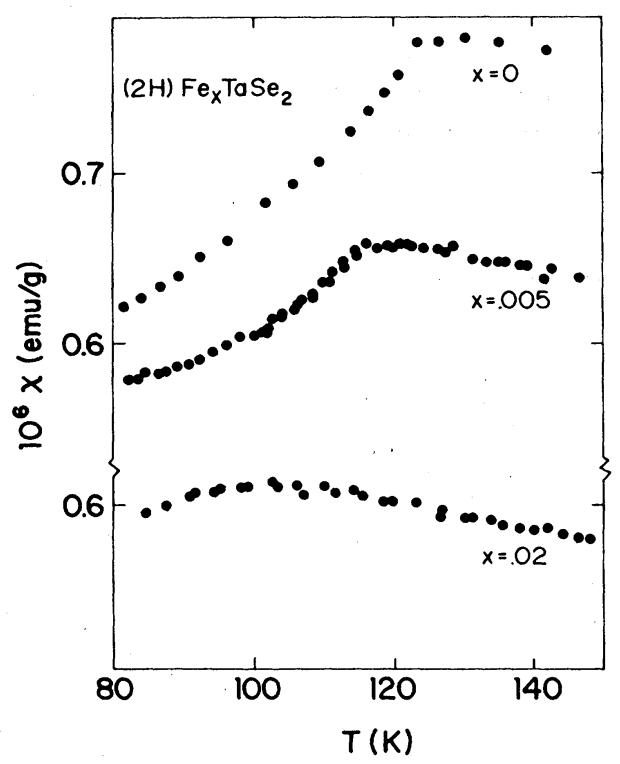

FIG. 7. Measured susceptibility vs temperature for $2 \mathrm{H}$ $\mathrm{Fe}_{x} \mathrm{TaSe}_{2}$ with $x=0,0.01,0.02$. The CDW transition is depressed by $\sim 20 \mathrm{~K}$ at $x=0.02$. 


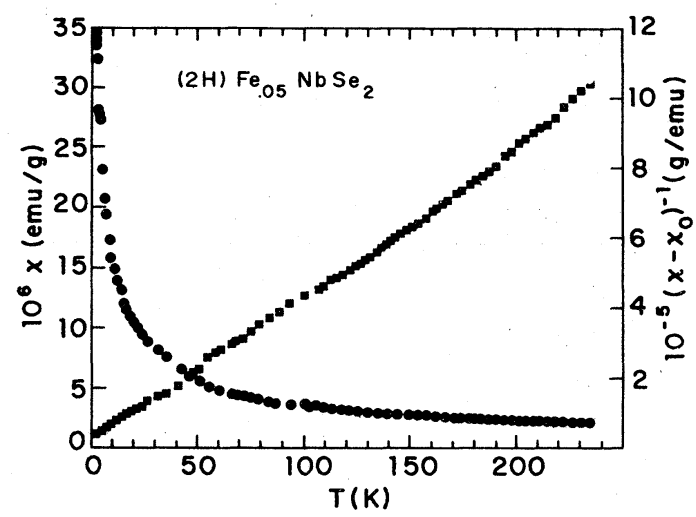

FIG. 8. Susceptibility as a function of temperature for $2 \mathrm{H}-\mathrm{Fe}_{0,05} \mathrm{NbSe}_{2}$. A Curie-Weiss behavior is observed but no maximum is present down to $\sim 1.5 \mathrm{~K}$.

The susceptibility maximum occurs at progressively higher temperatures as the Fe concentration is increased in the $2 H-\mathrm{Fe}_{x} \mathrm{TaSe}_{2}$ crystals. The value of the Curie constant also increases with $\mathrm{Fe}$ concentration and the systematic variation of the Curie constant is probably a good check on the relative Fe concentration in a given crystal. The relation between the susceptibility maximum and the Curie constant is shown in Fig. 6 and gives a smooth correlated behavior over the range $x=0.02-0.10$.

At low $\mathrm{Fe}$ concentrations the susceptibility is also influenced by the presence of the CDW. For $x$ between 0 and 0.02 the CDW onset is depressed in temperature and broadened as shown in Fig. 7. At $x=0.02$ the onset is depressed by $\sim 20 \mathrm{~K}$, but the CDW still has a substantial effect on the susceptibility below $100 \mathrm{~K}$ as seen in the lowest curve of Fig. 7 . For crystals with higher Fe concentrations the susceptibility is dominated in this temperature range by the rapidly increasing contribution from the $\mathrm{Fe}$ and the CDW onset cannot be resolved. However, the Curie-Weiss plot deviates from a straight line below $100 \mathrm{~K}$ and this may indicate a contribution from a CDW. The Curie-Weiss analysis has not been corrected for contributions from the $\mathrm{CDW}$ and this may play a role in the variation of $p_{\text {eff }}$ and $\theta$ as a function of $\mathrm{Fe}$ concentration.

The $\theta$ values become progressively more positive at higher iron concentrations (see Table I). This can be the result of SDW formation and can also result from the formation of a "spin-glass"-type magnetic state. This will be discussed in more detail in Sec. IV.

\section{B. Susceptibility of $2 \mathrm{H}-\mathrm{Fe}_{x} \mathrm{NbSe}_{2}$}

The pure $2 H$ phase of $\mathrm{NbSe}_{2}$ has a normal to incommensurate $\mathrm{CDW}$ transition at $\sim 35 \mathrm{~K}$ and the susceptibility of the excess $\mathrm{Fe}$ doped $2 \mathrm{H}$ phase of $\mathrm{NbSe}_{2}$ shows a Curie-Weiss behavior similar to that

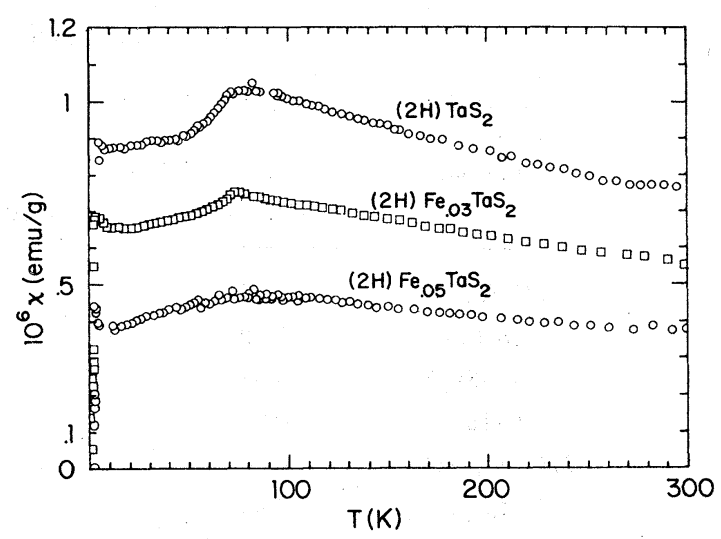

FIG. 9. Susceptibility as a function of temperature for $2 H-\mathrm{Fe}_{x} \mathrm{TaS}_{2}$. Iron concentrations corresponding to $x=0$, 0.03 , and 0.05 are shown. The CDW transition becomes smeared but the onset temperature is not reduced.

of $2 \mathrm{H}-\mathrm{Fe}_{x} \mathrm{TaSe}_{2}$. Both the $2 \mathrm{H}$ single crystals and powders give values of $p_{\text {eff }}$ in the range 3.0-3.2 as listed in Table I. A typical susceptibility plot as a function of temperature is shown in Fig. 8. The values of $\Theta$ are in the range -6 to $-7 \mathrm{~K}$ for $2 \mathrm{H}$ $\mathrm{Fe}_{0.05} \mathrm{NbSe}_{2}$ and do not show a trend toward positive values as observed for the higher $\mathrm{Fe}$ concentrations in $2 H-\mathrm{Fe}_{x} \mathrm{TaSe}_{2}$. In the case of $2 H-\mathrm{Fe}_{x} \mathrm{TaSe}_{2}$ increasing $\mathrm{Fe}$ concentration clearly drives $\Theta$ positive due to the transition associated with the possible SDW onset. Such a transition if present in $2 \mathrm{H}-\mathrm{Fe}_{x} \mathrm{NbSe}_{2}$ is much weaker and has not been observed down to the lowest measuring temperature of $\sim 1.5$.

\section{Susceptibility of $2 \mathrm{H}-\mathrm{Fe}_{x} \mathrm{TaS}_{2}$}

The pure $2 \mathrm{H}$ phase of $\mathrm{TaS}_{2}$ has a normal to commensurate CDW transition ${ }^{15}$ at $\sim 75 \mathrm{~K}$. The magnetic susceptibility of $2 \mathrm{H}-\mathrm{TaS}_{2}$ crystals with excess Fe concentrations in the range $0-5$ at. $\%$ has been measured in the temperature range $1-300 \mathrm{~K}$. The results are shown in Fig. 9 for $\mathrm{Fe}$ concentrations of 0,3 , and 5 at. \%. In the pure $2 \mathrm{H}-\mathrm{TaS}_{2}$ crystal the CDW transition is clearly observed as indicated by the onset of a decreasing susceptibility below $75 \mathrm{~K}$. The $2 \mathrm{H}$ $\mathrm{Fe}_{0.03} \mathrm{TaS}_{2}$ crystal also shows a strong susceptibility decrease below $75 \mathrm{~K}$ and indicates that the $\mathrm{Fe}$ doping while lowering the susceptibility over the entire temperature range has not suppressed the CDW transition. The $2 H-\mathrm{Fe}_{0.05} \mathrm{TaS}_{2}$ also shows a substantial drop in susceptibility below $75 \mathrm{~K}$ although the CDW transition appears to be smeared in the higher concentration crystals. The rapid drop at the lowest temperatures for the 3 and 5 at. \% crystals results from the transition to superconductivity below $3.5 \mathrm{~K}$. Numerical values of $X$ are listed in Table II.

No evidence of magnetic-moment formation is ob- 
TABLE II. Susceptibilities of nonmagnetic layer materials.

\begin{tabular}{clccc}
\hline \hline Material & $\begin{array}{c}x_{\perp}(300 \mathrm{~K}) \\
\left(10^{-6} \mathrm{emu} / \mathrm{g}\right)\end{array}$ & $\begin{array}{c}x_{\perp}(4.2 \mathrm{~K}) \\
\left(10^{-6} \mathrm{emu} / \mathrm{g}\right)\end{array}$ & $\begin{array}{c}x_{11}(300 \mathrm{~K}) \\
\left(10^{-6} \mathrm{emu} / \mathrm{g}\right)\end{array}$ & $\begin{array}{c}x_{11}(4.2 \mathrm{~K}) \\
\left(10^{-6} \mathrm{emu} / \mathrm{g}\right)\end{array}$ \\
\hline $4 H b-\mathrm{TaSe}_{2}{ }^{\mathrm{a}}$ & $0.28 \pm 0.05$ & & $0.10 \pm 0.05$ & \\
$4 H b-\mathrm{TaS}_{2}$ & 0.78 & & 0.26 & 0.26 \\
$2 H-\mathrm{TaSe}_{2}{ }^{\mathrm{b}}$ & 0.68 & 0.56 & 0.29 & 0.35 \\
$2 H-\mathrm{TaS}_{2}$ & 0.83 & 0.89 & 0.36 & \\
$2 H-\mathrm{Fe}_{0.03} \mathrm{TaS}_{2}$ & 0.60 & & 0.30 & \\
$2 H-\mathrm{Fe}_{0.05} \mathrm{TaS}_{2}$ & 0.40 & & 0.15 & \\
\end{tabular}

${ }^{a}$ F. J. DiSalvo, D. E. Moncton, J. A. Wilson, and S. Mahajan, Phys. Rev. B 14, 1543 (1976).

bJ. L. Benchimol, F. T. Hedgecock, and F. J. DiSalvo, Solid State Comm. 25, 677 (1978).

served in the susceptibility measurements on $2 \mathrm{H}$ $\mathrm{Fe}_{x} \mathrm{TaS}_{2}$ single crystals for the $\mathrm{Fe}$ concentration range of 5 at. $\%$ and below. In general the measurements indicate a weakening of the $\mathrm{CDW}$ in the Fe-doped crystals with an accompanying rise in the superconducting transition temperature while the CDW onset temperature remains approximately constant as a function of Fe concentration.

\section{Susceptibility of $4 H b-\mathrm{Fe}_{x} \mathrm{TaS}_{2}$ and $4 H b-\mathrm{Fe}_{x} \mathrm{TaSe}_{2}$}

The pure $4 \mathrm{Hb}$ phases of $\mathrm{TaS}_{2}$ and $\mathrm{TaSe}_{2}$ show two CDW transitions, one at relatively high temperature associated with the octahedral layers and one at lower temperature associated with the trigonal prismatic layers. The onset temperatures are $\sim 315$ and $20 \mathrm{~K}$ for the $4 \mathrm{Hb}$ - $\mathrm{TaS}_{2}$ crystals $^{16}$ and $\sim 600$ and $75 \mathrm{~K}$ for the $4 \mathrm{Hb}-\mathrm{TaSe}_{2}$ crystals. $^{17}$ The $4 \mathrm{Hb}$ phase of $\mathrm{TaS}_{2}$ can be grown with up to at least 5 at. $\%$ excess $\mathrm{Fe}$ concentration and the CDW transitions are not evident in the susceptibility of the crystals with 5 at. \% Fe. The susceptibilities have been measured for fields both parallel and perpendicular to the layers and representative plots are shown in Fig. 10 for a $4 \mathrm{Hb}$ $\mathrm{Fe}_{0.05} \mathrm{TaS}_{2}$ crystal. The susceptibility rises rapidly at low temperature and reaches a maximum just below $10 \mathrm{~K}$. At lower temperatures a rapid drop is observed indicating a magnetic ordering similar to that observed in $2 \mathrm{H}-\mathrm{Fe}_{x} \mathrm{TaSe}_{2}$. At temperatures near the susceptibility maxima the anisotropy $\chi_{\perp} / \chi_{\|}$is approximately 2 . The susceptibility maximum is nearly field independent in the range $1-5 \mathrm{kG}$ as shown in Fig. 11 and at very low fields the maximum sharpens to a more cusplike behavior similar to that observed for $2 \mathrm{H}-\mathrm{Fe}_{0.08} \mathrm{TaSe}_{2}$ at low magnetic fields (see Fig. 5).

The higher temperature data fit a Curie-Weiss plot as also shown in Fig. 10 for fields parallel and per- pendicular to the layers. Calculated values of $p_{\text {eff }}$ are $5.0(\|)$ and $5.2(\perp)$, respectively. The values of $\theta$ are positive and equal to $1.8(\|)$ and $11(\perp)$. See Table III.

The $4 \mathrm{Hb}$ phase of $\mathrm{TaSe}_{2}$ is limited to a maximum $\mathrm{Fe}$ concentration of -2 at. \%. Above this concentration only the $2 H$ or the $1 T$ phase can be grown in the various temperature ranges. The crystals of $4 \mathrm{Hb}$ $\mathrm{Fe}_{0.02} \mathrm{TaSe}_{2}$ show only a very weak-local-moment formation and analysis of the Curie-Weiss plot gives a value of $p_{\text {eff }}=0.47$. This is an order of magnitude smaller than observed for most of the phases showing a strong-local-moment behavior and no evidence of susceptibility maxima or transport anomalies is observed. In an earlier experiment ${ }^{3}$ the susceptibility of a slowly cooled crystal showed a large anisotropy with the perpendicular $p_{\text {eff }}=4.4$ vs $p_{\text {eff }}=0.4$ parallel to the layers. Subsequent measurements on rapidly quenched crystals show only the low value of $p_{\text {eff }}$ for

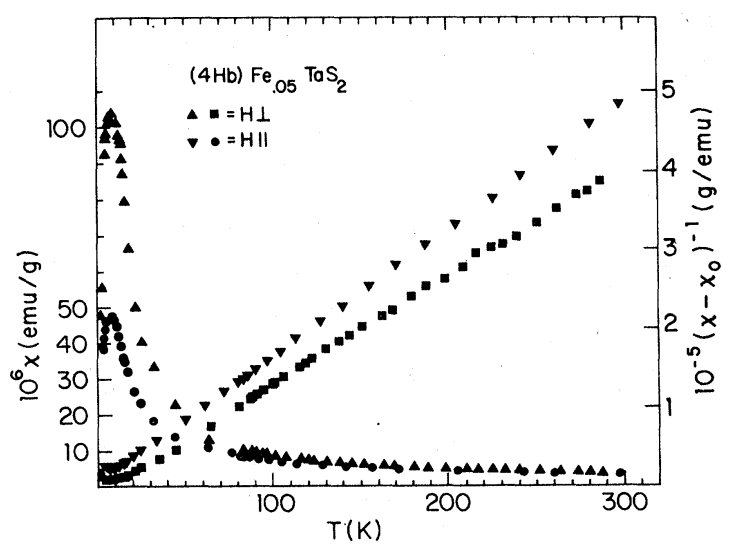

FIG. 10. Susceptibility as a function of temperature for $4 \mathrm{Hb}-\mathrm{Fe}_{0.05} \mathrm{TaS}_{2}$. A Curie-Weiss behavior is observed and a susceptibility maximum occurs at $7.5 \mathrm{~K}$. 
TABLE III. Susceptibility data for $4 H b-\mathrm{Fe}_{x} M X_{2}$ compounds.

\begin{tabular}{lcccc}
\hline \hline Material & Phase & $\begin{array}{c}p_{\text {eff }} \\
\left(\mu_{\mathrm{B}}\right)\end{array}$ & $\begin{array}{c}\Theta \\
(\mathrm{K})\end{array}$ & $\begin{array}{c}T_{\max } \\
(\mathrm{K})\end{array}$ \\
\hline $\mathrm{Fe}_{0.02} \mathrm{TaSe}_{2}$ & Mixed phase & $4.4_{\perp} \pm 0.2_{\perp}$ & $-5 \pm 1$ & \\
& (crystal) & $0.4_{\|}$ & 0 & \\
$\mathrm{Fe}_{0.02} \mathrm{TaSe}_{2}$ & $4 \mathrm{Hb}$ (crystal) & $0.8_{\perp}$ & -1.4 & \\
$\mathrm{Fe}_{0.05} \mathrm{TaS}_{2}$ & $4 \mathrm{Hb}$ (crystal) & $5.2_{\perp}$ & 11 & $7.5(4.5 \mathrm{kG})$ \\
& & $5.0_{\|}$ & 1.8 & $7.3(4.5 \mathrm{kG})$ \\
\hline
\end{tabular}

fields both parallel and perpendicular to the layers. The previous anisotropy may have been connected with a slight phase mixture or other anomaly occurring in the more slowly cooled crystal.

\section{E. Hall effect}

The Hall effect has been measured in all of the iron-doped compounds and phases reported in this work and the main features are outlined below. The pure phases have negative Hall coefficients at temperatures below the $\mathrm{CDW}$ transition while the $\mathrm{Fe}$ doped crystals showing a strong magnetic moment have positive Hall coefficients. The $2 H-\mathrm{Fe}_{x} \mathrm{TaSe}_{2}$ crystals exhibit positive Hall coefficients which show strong nonlinear magnetic contributions at low fields as shown in the upper curves of Fig. 12. This nonlinear magnetic contribution reaches a maximum at

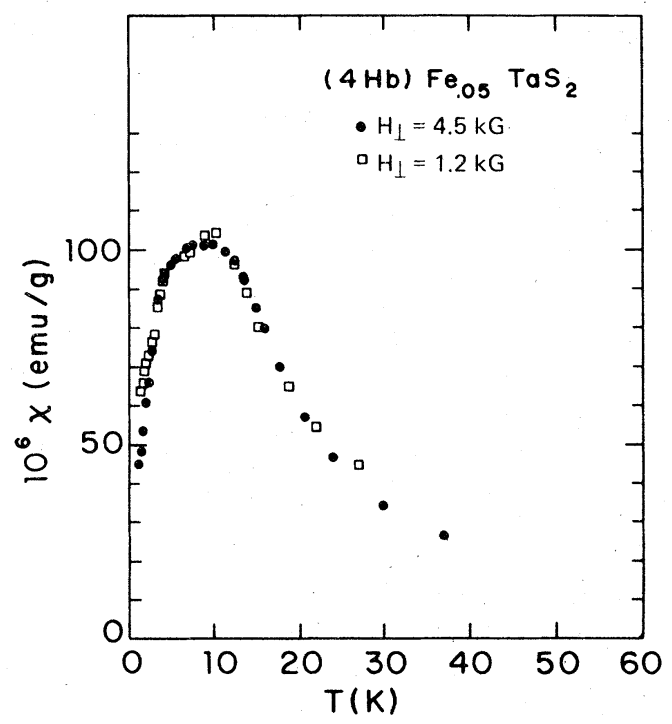

FIG. 11. Field dependence of the susceptibility maximum in $4 \mathrm{Hb}-\mathrm{Fe}_{0.05} \mathrm{TaS}_{2}$. In the range $1-5 \mathrm{kG}$ very little field dependence is observed and the magnetic state is quite stiff for applied fields in this range. $x=0.05$ and has decreased substantially at $x=0.10$. The field dependence is logarithmic at high fields and has previously been analyzed ${ }^{1}$ using expressions for magnetic scattering developed by Béal-Monod and Weiner. ${ }^{18}$ The nonlinear field dependence is also highly temperature dependent and increases rapidly below $\sim 20 \mathrm{~K}$ as shown in Fig. 13. In the case of $4 H b-\mathrm{Fe}_{x} \mathrm{TaSe}_{2}$ the low-temperature Hall coefficient is still negative, but is reduced in magnitude from that of the pure phase as shown in the lower three curves of Fig. 12. No magnetic contribution is observed at low fields and the behavior is consistent with the absence of anomalies in the resistance and magnetoresistance.

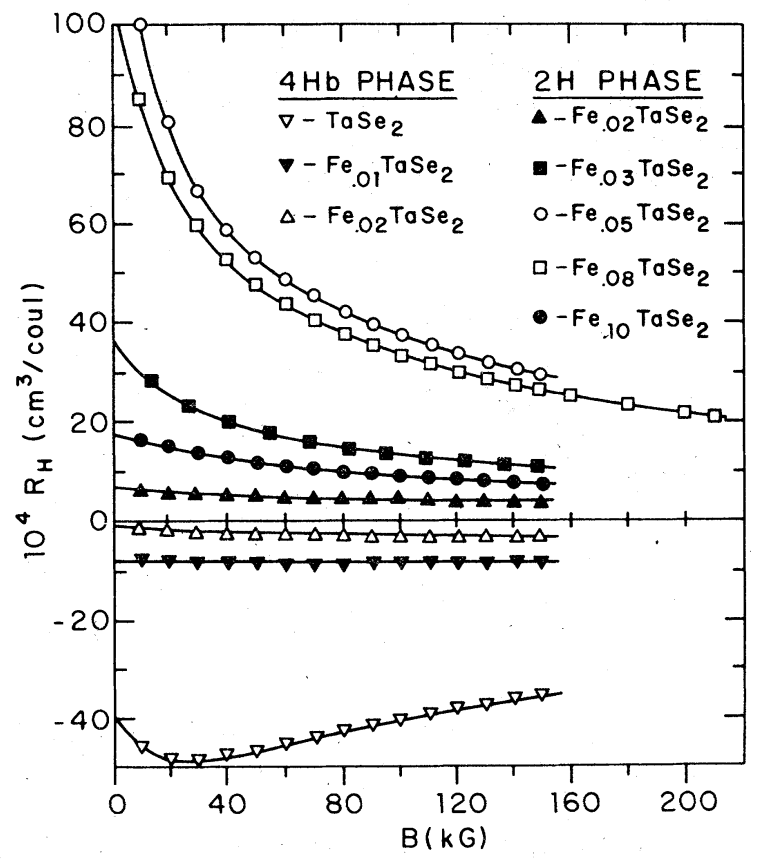

FIG. 12. Hall coefficient vs magnetic field at $4.2 \mathrm{~K}$ for $2 H$ - $\mathrm{Fe}_{x} \mathrm{TaSe}_{2}$ and $4 H b-\mathrm{Fe}_{x} \mathrm{TaSe}_{2}$. For the $2 H$ phase coefficients are positive and show a very nonlinear behavior. For the $4 H b$ phase coefficients are negative and a very small field dependence is observed. 


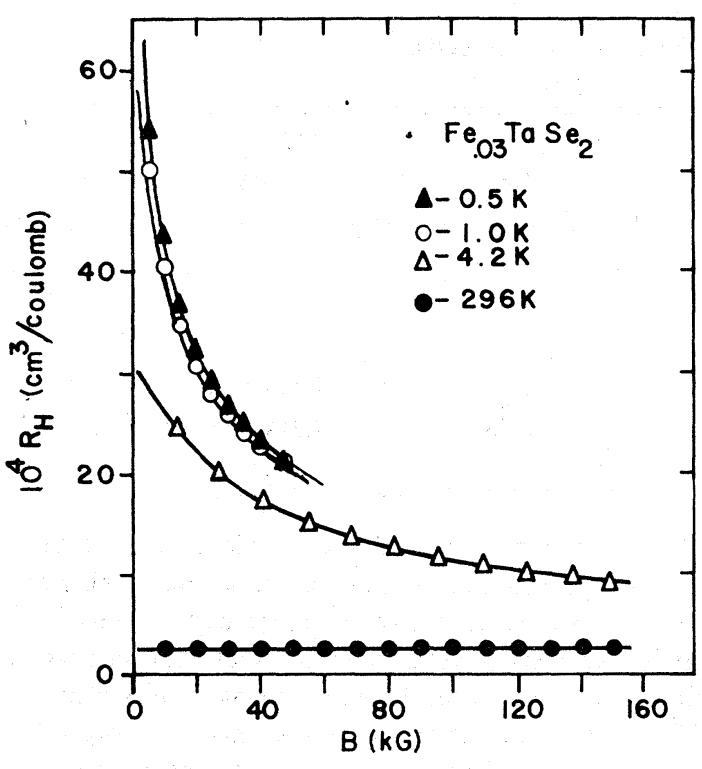

FIG. 13. Nonlinear Hall coefficient as a function of temperature in $2 \mathrm{H}-\mathrm{Fe}_{0.03} \mathrm{TaSe}_{2}$. The nonlinear behavior decreases rapidly above $20 \mathrm{~K}$.

The Hall coefficient in $2 H-\mathrm{Fe}_{x} \mathrm{TaS}_{2}$ shows a positive to negative transition near the CDW onset as shown in Fig. 14. This is consistent with the susceptibility measurements and no magnetic behavior is indicated although the magnitude of the negative Hall coefficient has been reduced by the excess $\mathrm{Fe}$. In contrast the $4 \mathrm{Hb}$ - $\mathrm{Fe}_{x} \mathrm{TaS}_{2}$ again shows the positive nonlinear Hall coefficent associated with magnetic scattering.

The anomalous Hall coefficients which show a very

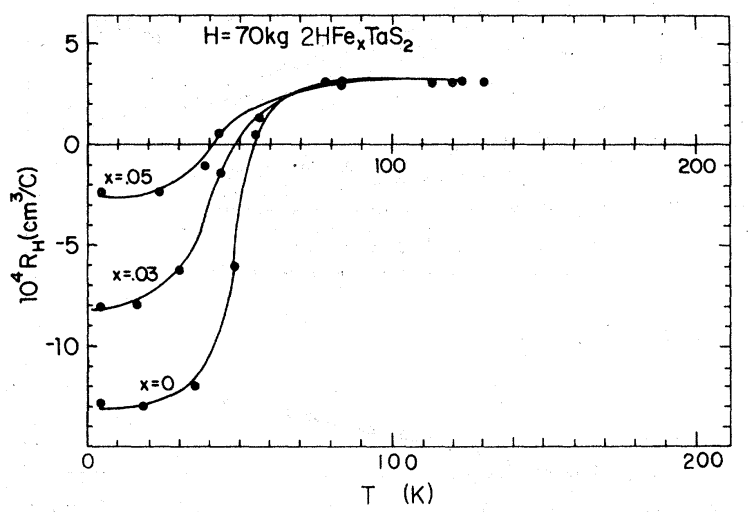

FIG. 14. Hall coefficient as a function of temperature in $2 H-\mathrm{Fe}_{x} \mathrm{TaS}_{2}$. The CDW transition corresponding to the positive to negative transition in the Hall coefficient is present in both iron-doped specimens. The onset of the CDW is not appreciably changed as was also observed in the susceptibility measurement. See Fig. 9. strong nonlinear dependence on applied magnetic field and temperature are predicted within the framework of SDW formation at low temperatures. The main points are summarized in Sec. IV and theoretical details can be found in an article by Antoniou. ${ }^{8}$

\section{DISCUSSION}

The susceptibility measurements on the layerstructure diselenides doped with excess iron have shown the formation of relatively strong local moments in some cases and very small or zero moments in others. $2 \mathrm{H}$ - $\mathrm{Fe}_{x} \mathrm{NbSe}_{2}, 2 \mathrm{H}-\mathrm{Fe}_{x} \mathrm{TaSe}_{2}$, and $4 \mathrm{Hb}$ $\mathrm{Fe}_{x} \mathrm{TaS}_{2}$ show large moments accompanied by magnetic behavior in the transport and susceptibility while $2 H-\mathrm{Fe}_{x} \mathrm{TaS}_{2}$ and $4 H b$ - $\mathrm{Fe}_{x} \mathrm{TaSe}_{2}$ show no magnetic anomalies. In the cases with magnetic moment formation the temperature-dependent susceptibilities follow a Curie-Weiss law of the form;

$$
\chi(T)=\chi_{0}+C /(T-\theta) .
$$

The Curie constant $C$ is generally related to an effective moment $p_{\text {eff }}$ by

$$
C=N_{i} p_{\text {eff }}^{2} \mu_{\mathrm{B}}^{2} / 3 k_{B},
$$

where $N_{i}$ is the impurity concentration, $\mu_{\mathrm{B}}$ the Bohr magneton, and $p_{\text {eff }}$ is related to the spin by

$$
p_{\text {eff }}=g[S(S+1)]^{1 / 2} \text {. }
$$

For $2 H-\mathrm{Fe}_{x} \mathrm{TaSe}_{2}$ with $x$ in the range $0.02-0.10$ calculations from the observed Curie constants give values of $p_{\text {eff }}$ in the range $2.5-4.5$ corresponding to spin values between 1 and 2 . While $p_{\text {eff }}$ values corresponding to simple spin values of $\frac{1}{2}, 1, \frac{3}{2}$, etc., are not required by the Friedel-Anderson localmoment picture the variations in the above numbers can result from several factors. The CDW is still present and can modify the susceptibility behavior particularly in the more dilute alloys. The onset temperature and strength of a possible SDW transition can also vary with iron concentration and can modify the Curie-Weiss plot with a corresponding change in $p_{\text {eff. }}$. These factors as well as fluctuations in the precise iron concentration can all contribute to apparent variations in $p_{\text {eff }}$ and the range of values obtained for different iron concentrations probably reflects a combination of factors.

The transport anomalies characterized by a resistance minimum, a negative magnetoresistance, a logarithmic temperature, and field dependence of the resistance all suggest a possible Kondo-type mechanism. Fits of the data to theoretical expressions of Béal-Monod and Weiner ${ }^{14}$ showed consistency with such a mechanism and were fully discussed in Ref. 1 . However the strong resistance and susceptibility maxima which were observed in the range $4-10 \mathrm{~K}$ for 
concentrations of excess iron up to 10 at. \% were not adequately explained by a Kondo-type mechanism.

Antoniou and Cohen ${ }^{7}$ have suggested the formation of a spin-density wave (SDW) as the principal mechanism leading to the maxima in resistance and susceptibility. The susceptibility maximum would occur at the onset of the SDW which forces a reduction in the magnetization. The resistance maximum would result from the increase in amplitude of the SDW with decreasing temperature and a corresponding reduction in the spin-flip scattering. The SDW formation would also play a role in the negative magnetoresistance and the nonlinear Hall effect.

For the iron-doped layer structures where a strong impurity moment is formed the normal to CDW transition temperature should be reduced and the transition smeared so that a sharp susceptibility maximum due to the CDW onset should no longer be observed. At the same time an aperiodic modification of a periodic magnetic distortion should be stabilized leading to a rapid increase of susceptibility followed by a maximum in the susceptibility at the SDW onset. These predicted features have been observed particularly well in $2 \mathrm{H}$ - $\mathrm{Fe}_{x} \mathrm{TaSe}_{2}$ and many of the detailed experimental results confirm the expected behavior.

The Antoniou-Cohen theory develops a model calculation of an impurity induced SDW in the background of a smeared CDW. The susceptibility maximum associated with the SDW onset should display a number of characteristic features: (i) The magnitude should decrease with increased applied magnetic field. (ii) $T_{\max }$ should decrease and the maximum become more rounded with increased magnetic fieid. (iii) There should be a pronounced asymmetry above and below the susceptibility maximum with the susceptibility dropping much more rapidly below $T_{\max }$. All of these features are observed as shown in the experimental curves of Figs. 2 and 5.

The detailed calculations can be found in a paper, by Antoniou ${ }^{8}$. They lead to a bulk high-temperature susceptibility given by

$\chi^{\prime}=\frac{1}{\rho h a_{2}}+\frac{\eta\left(1-J_{0} / a_{2}\right)^{2}}{a_{s} \rho h\left(T-\eta J_{0}^{2} / a_{2} a_{s}\right)}$,

where $\rho$ is the density of the material, $h$ is the height of a unit cell, and $\eta$ is the impurity area density. The constants $a_{2}$ and $a_{s}$ are parameters in the LandauGinzburg free energy associated with the uniform magnetization term and the impurity spin term, respectively. $J_{0}$ is a constant describing the coupling between the impurity spins and the spin density. This leads to a Curie-Weiss high-temperature susceptibility of the form

$$
\chi^{\prime}=\chi_{0}+\frac{C_{1}}{T-\Theta}
$$

with

$$
\begin{aligned}
& \chi_{0}=\frac{1}{\rho h a_{2}}, \\
& C_{1}=\eta\left(1-\frac{J_{0}}{a_{2}}\right)^{2}, \\
& \Theta=\frac{J_{0}^{2}}{a_{2} a_{s}} .
\end{aligned}
$$

The Curie constant can be rewritten

$$
C_{1}=\frac{N_{i} p_{\mathrm{eff}}^{2}}{3 k_{B} \rho}
$$

with

$$
p_{\text {eff }}=p_{0}\left|1-\frac{J_{0}}{a_{2}}\right|
$$

where

$$
p_{0}=\left(\frac{3 k_{B}}{a_{s}}\right)^{1 / 2}
$$

is the free impurity moment. This model predicts that $\theta$ should be positive and should increase with impurity concentration $\eta$. This is the general trend observed for $2 H-\mathrm{Fe}_{x} \mathrm{TaSe}_{2}$ as shown in Table I although at low concentrations small negative values are observed. The negative values may result from using plots uncorrected for the contribution from the CDW. The observed $p_{\text {eff }}$ values are lower than the value expected for the spin contribution from $\mathrm{Fe}^{2+}$ which should give $4.9 \mu_{\mathrm{B}}$. Therefore using Eq. (6b) $J_{0}$ should be positive corresponding to antiferromagnetic coupling between the impurity spins and the spin density of the conduction electrons.

The previous attempt to fit the transport data to an $s$ - $d$ exchange model for a Kondo-type interaction was consistent with an antiferromagnetic exchange constant although the present SDW model represents a quite different mechanism. The transport results can also be explained within the framework of the SDW formation although the resistance minimum may still result from a generalized Kondo-type mechanism. In the SDW model the extraordinary Hall coefficient should behave much like the bulk susceptibility with a strong nonlinear rise at low temperature. Applied fields should also reduce the Hall coefficient for temperatures above $T_{\max }$ while below $T_{\max }$ the nonlinear field reduction should be less pronounced. As shown in Fig. 12 the concentration dependence of the nonlinear field dependence of the Hall coefficient measured at $4.2 \mathrm{~K}$ for $2 \mathrm{H}-\mathrm{Fe}_{x} \mathrm{TaSe}_{2}$ reaches a maximum at $x=0.05$ and has decreased substantially at $x=0.10$. This is consistent with the expected rise of $T_{\max }$ to above $4.2 \mathrm{~K}$ as the $\mathrm{Fe}$ concentration is increased from $x=0.02-0.10$ 


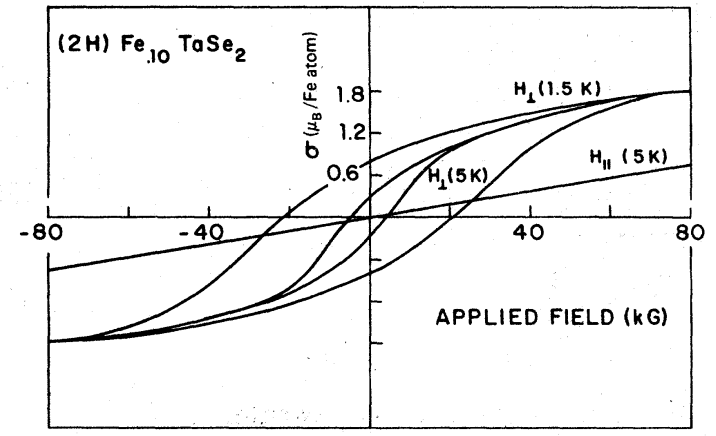

FIG. 15. Magnetization as a function of magnetic field measured at 1.5 and $5 \mathrm{~K}$. For field perpendicular to the layers a large temperature-dependent hysteresis is observed. For fields parallel to the layers a hysteresis is not observed.

The resistance and magnetoresistance behavior can also be explained within the context of an SDW model. The negative magnetoresistance can result from a suppression of spin-flip scattering due either to the application of an external field with spin alignment as occurs in Kondo-type systems and to the onset of an internal field which would lock the impurity spins in fixed directions. The interplay between the external field and the internal fields created by the SDW can produce a number of detailed changes in the magnetoresistance and these have not been examined by experiment with sufficient detail as yet. However, initial results reported in Ref. 1 indicate that changes in the magnitude of the negative magnetoresistance above and below the onset temperature of the SDW follow the predicted behavior.

At higher concentrations of excess $\mathrm{Fe}$ the $2 \mathrm{H}$ $\mathrm{Fe}_{x} \mathrm{TaSe}_{2}$ crystals develop a pronounced hysteresis as shown in Fig. 15 for a crystal with $x=0.10$. The field is applied perpendicular to the layers and the hysteresis develops rapidly below $T_{\max }$ which is -13 $\mathrm{K}$ in this case. Both the hysteresis and the susceptibility maximum are similar to features observed in spin-glass systems ${ }^{19}$ which are described as systems of interacting impurity spins where the spins "freezeout" in random directions below a certain critical temperature. The random impurity moment interaction is in this case mediated by the electron gas through the RKKY (Ruderman-Kittel-Kasuya-Yosida) interaction and in zero field the random phase of this interaction results in no net magnetic moment formation. However, the "freeze-out" of the random moments produces remanance, irreversibility, and relaxation and at higher concentrations short-range order develops giving rise to magnetic clusters which contribute to magnetic hysteresis. Above the "freeze-out" temperature the behavior is Curie-Weiss-like and for higher impurity concentrations the $\Theta$ values are positive due to the short-range ordering.

The Antoniou-Cohen theory of SDW formation at present omits the random nature of the phase of the SDW due to the random spatial distribution of the impurity moments. They point out that including these effects would give rise to a type of spin-glass state which they call a "spin-density-wave glass" and that such a state would be expected to exhibit the characteristic hysteresis which is observed.

The experimental results are at present generally consistent with the behavior expected for either the SDW induced glass or the more conventional spin glass. However, the fact that the susceptibility maxima are not very sensitive to magnetic fields in the range $1-5 \mathrm{kG}$ suggests a rather stiff magnetic system which may be more consistent with the SDW interaction. The layer-structure Fermi surfaces also show strong CDW transitions and the electronic structure should be favorable for SDW formation. However, further experiments including neutron-diffraction experiments will be necessary to clearly establish the type of magnetic state which forms in the Fe-doped layer crystals at low temperatures.

The result that dilute excess iron impurities in $2 \mathrm{H}$ $\mathrm{TaS}_{2}$ do not form moments is rather unusual. For $2 H-\mathrm{Fe}_{x} \mathrm{TaS}_{2}$ crystals with $x=0.28$ a ferromagnetic state ${ }^{20}$ is observed with $T_{c}=7.3 \pm 5^{\circ} \mathrm{K}$ and above $T_{c}$ a Curie-Weiss behavior is observed with $p_{\text {eff }}=4.5 \mu_{\mathrm{B}}$. The magnetic behavior therefore undergoes some type of transition for intermediate iron concentrations. This has not been examined experimentally as yet since the crystal-growth process does not yield good crystals at intermediate $\mathrm{Fe}$ concentrations due to phase mixtures or other instabilities.

The $2 \mathrm{H}$ phases of $\mathrm{TaSe}_{2}$ and $\mathrm{TaS}_{2}$ have essentially similar high-temperature band structures ${ }^{21}$ with only small differences in the undulations of the cylindrical Fermi surfaces. In the CDW state the Fermi surface of $2 \mathrm{H}-\mathrm{TaS}_{2}$ develops a group of Fermi-surface sections $^{9}$ with areas an order of magnitude smaller than observed in the CDW state 22,23 of $2 H-\mathrm{TaSe}_{2}$. This indicates a subtle difference between the two materials in the CDW state although the different sections of Fermi surface involve only $10^{-2}$ to $10^{-4}$ electrons per atom. The transition to the $4 H b$ phase of $\mathrm{TaS}_{2}$ does not significantly change the Fermi-surface sections $s^{9,22}$ attributed to the trigonal prismatic CDW state of the pure material but the dilute iron impurities now form strong moments with $p_{\text {eff }} \approx 5 \mu_{\mathrm{B}}$.

The formation of the magnetic impurity state depends on the position of the virtual $d$ "levels" relative to the Fermi level and on the width of these levels. Anderson ${ }^{24}$ has examined the general conditions leading to magnetic and nonmagnetic impurity states and has developed expressions for the relevant parameters. A transition from a magnetic to a nonmagnetic state can be caused by changes in the density of states or by motion of the Fermi level. Although the electron transfer from the octahedral to the trigonal prismatic layers in $4 \mathrm{Hb}-\mathrm{TaS}_{2}$ is estimated 
to be small ${ }^{9}$ the system can be quite sensitive to small shifts if conditions for the magnetic transition are closely balanced. Further and more detailed experiment and calculation will be needed to resolve the precise mechanisms. A general development for both CDW and SDW formation as well as mixed CDW and SDW systems has recently been given by Denley and Falicov. ${ }^{25}$

\section{CONCLUSIONS}

Measurements of the magnetic susceptibilities in layer-structure dichalcogenides doped with excess $\mathrm{Fe}$ in the range $0-10$ at. \% have been used to study local-moment formation and the resulting magnetic anomalies in the electronic properties. In addition to a Kondo-like resistance minimum and a CurieWeiss-like susceptibility induced by excess $\mathrm{Fe}$ impurities in $2 \mathrm{H}-\mathrm{TaSe}_{2}$ a strong susceptibility maximum is observed at low temperature. The temperature of the maximum scales with $\mathrm{Fe}$ concentration and reaches $\sim 13 \mathrm{~K}$ at 10 at. \%. An analysis of this maximum shows that it is consistent with the onset of a spindensity wave stabilized by the magnetic impurities. A number of the magnetic features are consistent with a spin-glass type of transition which can be induced by the spin-density-wave onset. The $\mathrm{Fe}$ impurities in $2 \mathrm{H}$-TaSe ${ }_{2}$ depress the charge-density-wave onset temperature while forming the local moments. Dilute $\mathrm{Fe}$ impurities in $2 \mathrm{H}-\mathrm{TaS}_{2}$ do not suppress the charge- density-wave onset temperature and local-moment formation is not observed. The local-moment formation and susceptibility maximum appears again in $4 \mathrm{Hb}-\mathrm{TaS}_{2}$ doped with excess $\mathrm{Fe}$ but is absent in $4 \mathrm{Hb}$ $\mathrm{TaSe}_{2}$ doped with excess $\mathrm{Fe}$. These variations show a very sensitive interplay between local-moment formation, charge-density-wave depression, and the electronic structure of the particular layer compound and phase. The possible spin-density-wave onset needs further investigation, but the low-temperature magnetic transition is clearly a distinct new electronic state of these layer structure materials.

\section{ACKNOWLEDGMENTS}

The research has been supported at the University of Virginia by the U.S. DOE and at the University of Nebraska by the NSF. High magnetic field work in the range $80-200 \mathrm{kG}$ was performed while the authors were guests at the Francis Bitter National Magnet Laboratory, which is supported at the Massachusetts Institute of Technology by the NSF. The authors would like to thank P. D. Antoniou and Professor M. H. Cohen for many valuable discussions and for communication of their results prior to publication. Useful discussions have also been held with Professor L. M. Falicov, Professor M. Fowler, and Professor V. Celli. Estelle Phillips has contributed to the crystal-growth program and Dr. D. A. Whitney has contributed to the transport measurements.
${ }^{1}$ D. A. Whitney, R. M. Fleming, and R. V. Coleman, Phys. Rev. B 15, 3405 (1977).

${ }^{2}$ D. A. Whitney, R. M. Fleming, and R. V. Coleman, Solid State Commun. 18, 309 (1976)

${ }^{3}$ R. V. Coleman, R. M. Fleming, D. A. Whitney, E. R. Domb, and D. J. Sellmyer, AIP Conf. Proc. 29, 400 (1976).

${ }^{4}$ R. C. Morris, B. W. Young, and R. V. Coleman, AIP Conf. Proc. 18, 292 (1974).

${ }^{5}$ Robert M. Fleming and R. V. Coleman, Phys. Rev. Lett. 34, 1502 (1975).

${ }^{6}$ J. A. Wilson, F. J. DiSalvo, and S. Mahajan, Adv. Phys. 24, 117 (1975).

${ }^{7}$ P. D. Antoniou and Morrel H. Cohen, Bull. Am. Phys. Soc. 23, 246 (1978).

${ }^{8}$ P. D. Antoniou, Phys. Rev. (to be published)

${ }^{9}$ S. J. Hillenius and R. V. Coleman, Phys. Rev. B $\underline{18}, 3790$ (1978).

${ }^{10}$ J. M. Van den Berg and P. Crossee, Inorg. Chem. Acta $\underline{2}$, 143 (1968)

${ }^{11}$ E. Eibschütz, F. J. DiSalvo, G. W. Hull, Jr., and S. Mahajan, Appl. Phys. Lett. 27, 464 (1975).

${ }^{12}$ B. L. Morris and A. Wold, Rev. Sci. Instrum. 39, 1937 (1968).
${ }^{13}$ R. C. Morris, Phys. Rev. Lett. 34, 1164 (1975).

${ }^{14}$ D. E. Moncton, J. D. Aye, and F. J. DiSalvo, Phys. Rev. Lett. 34,734 (1975).

${ }^{15}$ J. P. Tidman, O. Singh, A. E. Curzon and R. F. Frindt, Philos. Mag. 30, 1191 (1974).

${ }^{16}$ F. J. DiSalvo, B. G. Bagley, J. M. VooHoeve, and J. V. Waszczak, Phys. Chem. Solids 34, 1357 (1973).

${ }^{17}$ F. J. DiSalvo, D. E. Moncton, J. A. Wilson, and S. Mahajan, Phys. Rev. B 14, 1543 (1976).

${ }^{18}$ M. T. Béal-Monod and R. A. Weiner, Phys. Rev. 170,552 (1968).

19J. D. Mydosh, AIP Conf. Proc. 24, 131 (1974).

${ }^{20}$ M. Eibschütz, F. J. DiSalvo, G. W. Hull, Jr., and S. Mahajan, Appl. Phys. Lett. 27, 464 (1975).

${ }^{21} \mathrm{G}$. Wexler and A. M. Wooley, J. Phys. C 9, 1185 (1976).

${ }^{22}$ R. M. Fleming and R. V. Coleman, Phys. Rev. B 16,302 (1977).

${ }^{23}$ J. E. Graebner, Solid State Commun. 21, 353 (1977).

${ }^{24} \mathrm{P}$. W. Anderson, Phys. Rev. 124, 41 (1961).

${ }^{25}$ D. Denley and L. M. Falicov, Phys. Rev. B 17, 1289 (1978). 